\title{
Review of: "Utilization of bitumen modified with pet bottles as an alternative binder for the production of paving blocks"
}

\author{
Valeria Loise $^{1}$ \\ 1 University of Calabria
}

Potential competing interests: The author(s) declared that no potential competing interests exist.

At the beginning, I would like to thank the Editor for allowing me to refer this paper.

This paper is well structured and the idea behind it is good, on the whole. However, here are some considerations.

1. In the Introduction, authors state that "PET is the chemical name for polyester". This is not correct, in fact PET is only one type of polyester, but not all polyesters are PET.

2. The last sentence ("This modification has beeen carried out..... and life costs of pavements") of the introduction is too long and it is difficult to understend the flow.

Please, enter all units of measure in Table 2.

3. It would be useful to insert a paragraph explaining how the samples were prepared, for example how the bottles were added to the bitumen?

4. In paragraph 3.4 it could be useful to explain why the skid resistance decreases as the quantity of PET increases.

5. In paragraph 3.4, there is a sentence, "TRRL Road Note 27", that is not connected to anything. 